\title{
Why is the propagation velocity of a photon in a transparent medium reduced?
}

\author{
Bart G. de Grooth \\ Department of Applied Physics, University of Twente, P.O. Box 217, 7500 AE Enschede, The Netherlands
}

(Received 19 June 1996; accepted 18 June 1997)

\begin{abstract}
A path integral formalism is used to describe the propagation of photons through a transparent medium. It is shown that the reduced phase velocity of light can be understood quantitatively by taking into account the contribution of all the possible classical paths the photon could have taken in order to reach a detector. These paths include all the multiple scattering processes by the atoms in the medium. (C) 1997 American Association of Physics Teachers.
\end{abstract}

\section{INTRODUCTION}

The work presented in this article was triggered by question No. 21 in the July 1995 issue of this journal, asking how Snell's law could be understood from a quantum mechanical point of view. Or, to quote the final sentence of this question: "Is there an easy way to see in quantum mechanics that light might be expected to travel at different speeds in different media." Once this is understood, Snell's law is a consequence. The qualitative answer to this question was given by Feynman in his masterpiece "QED: The strange world of atoms and photons.', 2 This book was written for the layman and although it does not contain a single formula, it explains all the essentials of physical optics, including the use of complex phasor algebra (introduced purely graphically) and a quantum interpretation of these phenomena. Reading this book gives physicists the feeling that they somehow have missed some basic literature on the quantum mechanical interpretation of common optical phenomena, but after a literature search we must conclude that this is not the case: The ideas described by Feynman are his personal interpretation of well-known phenomena. The views he presented are consistent with his famous path integral formulation, but now applied to the propagation of photons. In this view the probability of detecting a photon can be found from the analysis of all the possible alternative classical paths the photon could have taken to reach the detector. Apart from certain places in his Lectures on Physics, ${ }^{3}$ I have not found a consistent description of this sort in the literature.

Feynman was fascinated by the phenomena of propagation 
of light in a refractive medium quite early in his scientific career. As J. A. Wheeler ${ }^{4}$ remembered: ... and how could we understand, in terms of scattering and nothing but scattering, the propagation of a photon through a medium of variable refraction index... How many wonderful aspects of physics came together (in this enterprise): ... refractive index as a cumulative consequence of many individual scattering processes; spirals-Cornu and other-as a tool to add up scattered waves; and as a motto to inspire us, the phrase "everything as scattering". What fun it was... That work never got published but both of us went on in postwar years to capitalize on the insights we had won from it.

In this article I give a quantitative description of some of the ideas presented by Feynman. ${ }^{2}$ The main purpose is to describe the propagation of photons through a transparent medium in terms of scattering amplitudes. In essence it turns out to be a reinterpretation of the treatment of this problem given by M. B. James and D. J. Griffiths, ${ }^{5}$ who used a perturbative approach to analyze the polarization of the medium by an electric field. The reader is advised to consult this article for a comparison. I will start by stating the basic assumptions of the path integral formalism applied to the propagation of a photon.

\section{BASIC ASSUMPTIONS}

The approach followed in this paper is based upon the path integral formalism described in detail in the classic book of Feynman and Hibbs. ${ }^{6}$ It is necessary, however, to postulate an expression for the propagation of a single photon which cannot be found in the standard literature on path integrals. We will make the following basic assumptions.

(1) When light is detected it is manifest as a discrete particle with energy $\hbar \omega$ where $\omega$ is the frequency of the photon.

(2) A photon always moves with the speed of light $c$.

(3) The probability of detecting a photon at time $t$ at position $\mathbf{r}$ is proportional to $A(\mathbf{r}, t) . A^{*}(\mathbf{r}, t)$, where $A(\mathbf{r}, t)$ is a complex number called the detection amplitude.

(4) If there are different alternative ways (paths) that a photon could have taken in order to arrive at position $\mathbf{r}$ at time $t$ and it is impossible to determine, in principle, which of these paths the photon has actually taken without disturbing the outcome of the experiment, we find the amplitude $A(\mathbf{r}, t)$ by summing up all the amplitudes $A_{p}(\mathbf{r}, t)$ belonging to possible paths $p$. This is the case of "indistinguishable alternatives.',

(5) If there are different alternative ways (paths) that a photon could have taken in order to arrive at position $\mathbf{r}$ at time $t$ but it is possible to determine in principle which of these alternatives the photon has actually taken without disturbing the outcome of the experiment, we find the probability of detecting the photon by summing up all the probabilities belonging to possible paths $p$.

(6) If a particular path can be divided into a number of successive paths or events, the resulting amplitude for this path is equal to the product of the amplitudes of the successive paths or events.

(7) If a photon travels from position $Q$ to position $R$ and the amplitude to be at $Q$ at time $t$ is known to be $A_{Q}(t)$, then the amplitude associated with a particular path going from $Q$ to $R$ is proportional to $A_{Q}(t-s / c)$, where $s$ is the length of this path. The proportionality constant has to be chosen such that the total probability of detecting photons remains constant.

It is important to note that although we will talk about possible paths that a photon has taken, the essence is that as long as there is no way of telling which path the photon actually has taken, we can best imagine that the photon has taken all the paths simultaneously. In other words, the photon as a well-defined discrete particle is only manifest at the moment it is actually destroyed by measuring it with a detector. For the remainder of this article we will consider the case of a simple monochromatic light source for which, in terms of conventional electromagnetic field theory, the electric field in vacuum can be approximated by a plane wave propagating along the $x$ axis:

$$
E=E_{0} \cos (k x-\omega t),
$$

where $k$ is the wave vector, which is equal to $\omega / c$. For convenience we have chosen the phase to be zero for $t=0$ at $x=0$.

The intensity of this wave is given by the magnitude of the Poynting vector:

$$
I=\frac{\epsilon_{0} c}{2} E_{0}^{2} .
$$

(8) We now postulate that for this situation, where the light propagates without any disturbances, the amplitude for detecting a photon at position $x, y, z$ at time $t$ is given by

$$
A_{0}(x, t)=A e^{i(k x-\omega t)} .
$$

The central rule of the path integral formalism states that the amplitude of a certain path is proportional to $e^{(i / \hbar) S_{\text {path }}}$ where $S_{\text {path }}$ is the action given by ${ }^{6}$

$$
S_{\text {path }}=\int_{\text {path }}(T-V) d t
$$

and $T$ and $V$ are the kinetic and potential energy, respectively. For the propagation of a photon we have in effect taken $T=\hbar \omega$ (postulate 1) and $V=0$ so that with $k=\omega / c$ and $d t=d s / c$ for each path [postulate (2)], the amplitude for a certain path of length $s$ is proportional to $e^{i k s}$, in agreement with postulates (7) and (8). Thus the action is proportional to the path length and, because of postulate (2), also to the traveling time.

The amplitudes are now normalized so that the probability that a photon will pass through an area $Q$ during a time $d t$ is given by

$$
P=A^{2} O d t \text {. }
$$

Therefore, the light intensity is given by

$$
I=A^{2} \hbar \omega \text {. }
$$

By comparison, we find a relation between $A$ and $E_{0}$ :

$$
A=\left[\frac{c \epsilon_{0}}{2 \hbar \omega}\right]^{1 / 2} E_{0} .
$$

From this relation between $A$ and $E_{0}$ we see that the essence of our postulate (8) [Eq. (3)] is that the amplitude of detecting a photon is proportional to the electric field of Eq. (1) provided that it is written in complex notation [see Eq. (8) below]. To avoid complications that might obscure the essence of our approach we limit ourselves to situations in 


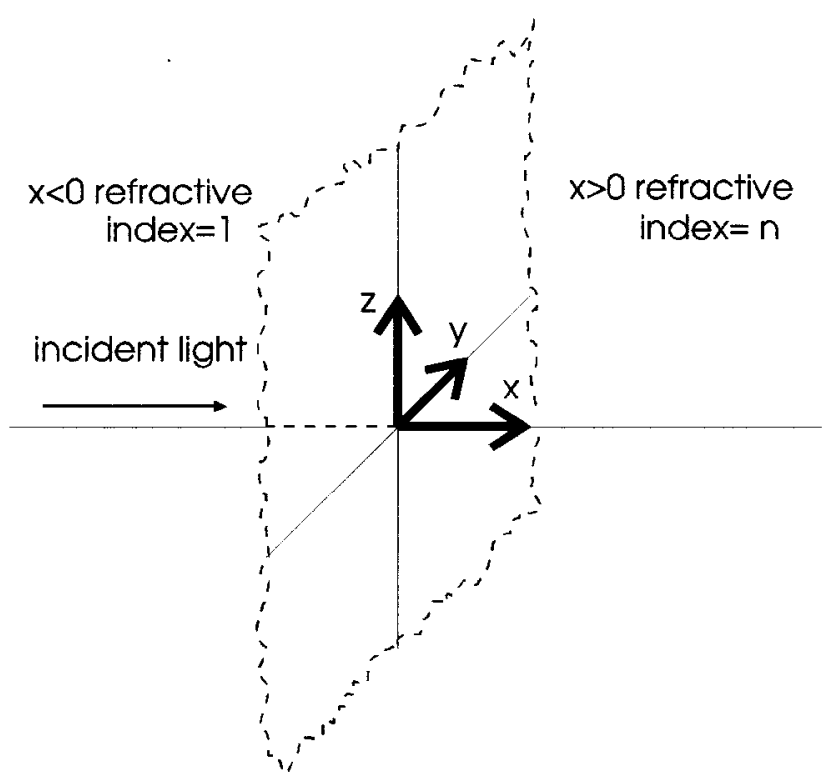

Fig. 1. A plane monochromatic beam of light originating from $x<0$ in vacuum is incident normally on a medium with refractive index $n$ at $x>0$.

which the polarization of light can be ignored so that we can use scalar notation. Generalization of these ideas to include polarization is relatively straightforward. For an extensive qualitative description of how these postulates lead to an understanding of diffraction theory I refer to Ref. 2.

\section{PHOTON PROPAGATION IN A TRANSPARENT MEDIUM OF INFINITE LENGTH}

The purpose of this section is to understand how the apparent velocity of light in a transparent medium is reduced. For this we consider the situation such that for $x<0$ we have vacuum and for $x>0$ space is filled with a homogeneous transparent dielectric medium with refractive index $n$ (Fig. $1)$. We will show that with our assumptions we can reproduce the well-known results for transmission and reflection of plane monochromatic light at normal incidence on a transparent medium.

\section{A. Electromagnetic approach}

We begin by recollecting the conventional description using electromagnetic field theory. ${ }^{7,8}$ Let the incoming light be described by the electric field propagating in the $x$ direction:

$$
E(x, t)=E_{0} e^{i(k x-\omega t)},
$$

where for convenience we use complex notation, where it is understood that from now on the physical electric field is given by the real part of $E$. The phase has been chosen to be zero for $t=0$ at $x=0$.

Let this field enter a medium at position $x=0$. Then the transmitted field at position $x$ in the medium is given by ${ }^{7}$

$$
E(x, t)=\frac{2}{n+1} E_{0} e^{i(n k x-\omega t)} .
$$

Thus the field propagates with a reduced (phase) velocity equal to $\omega / n k=c / n$. As we shall see later it turns out that it is more practical to work in terms of the electric susceptibility $\chi$ by using

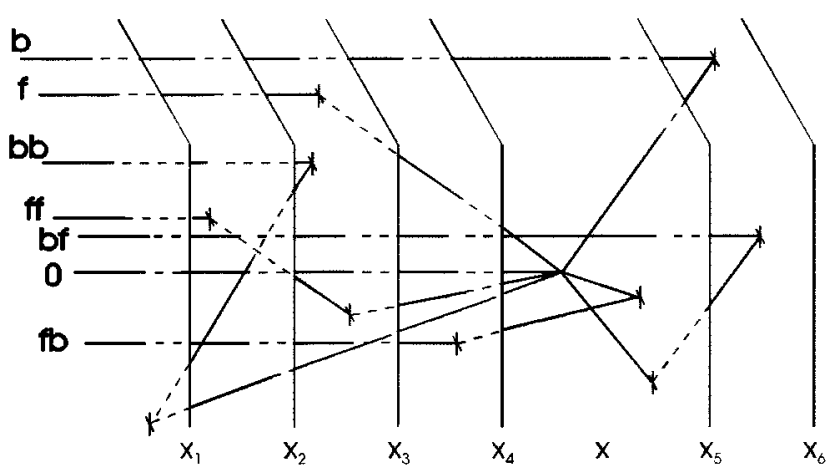

Fig. 2. In order to calculate the probability of detecting a photon at position $(x, 0,0)$ at time $t$, we have to consider all the different paths the photon could have taken. Generic paths up to second-order scattering are shown.

$$
n=\sqrt{1+\chi}
$$

Expanding Eq. (9) in terms of $\chi$ we can write

$$
\begin{aligned}
E(x, t)= & {\left[1+\left(-\frac{1}{4}+\frac{i k x}{2}\right) \chi+\left(\frac{1}{8}-\frac{i k x}{4}+\frac{(i k x)^{2}}{8}\right) \chi^{2}\right.} \\
& +\left(-\frac{5}{64}+\frac{5 i k x}{32}-\frac{3(i k x)^{2}}{32}+\frac{(i k x)^{3}}{48}\right) \chi^{3} \\
& \left.+O\left(\chi^{4}\right)\right] E_{0} e^{i(k x-\omega t)}
\end{aligned}
$$

In this way the electric field is mathematically rewritten as a sum of terms, for which each individual term apparently propagates with a (phase) velocity of $\omega / k=c$ ! It will now be shown that the terms in the expansion can be interpreted physically as originating from contributions of all the possible ways the photon could have taken in order to be detected at position $x$ at time $t$. The first term represents the amplitude that a photon has followed the direct path between light source and detector without any scattering. The second term will be shown to be due to the amplitude of all the possible paths where the photon has scattered once, the third term belongs to paths that contain double scattering, etc.

\section{B. Path integral formulation}

As discussed above, we now reinterpret the complex incoming electric field as being proportional to the amplitude for detecting a photon.

Suppose we detect a photon at position $(x, 0,0)$ in the medium $(x>0)$ at time $t$. In order to find the amplitude for this to occur, we have to sum up the amplitudes of all the possible ways the photon could have taken in principle from source to detector (see Fig. 2).

First we have the amplitude that the photon did not scatter at all. This is simply:

$$
A_{0}(x, t)=A e^{i(k x-\omega t)},
$$

the amplitude for a photon in vacuum [postulate (8)].

But the photon could also be emitted by the light source at some earlier time and be scattered at different positions before it reached the detector. Since under the condition that the light source is continuous there is no way of telling which of all the possible paths the detected photon actually has taken, we have to sum up all the amplitudes to be able to 
find the total amplitude to detect the photon [postulate (4)]. It is our job to find the amplitudes of all these processes and to add them up.

In order to do this, we first have to describe a single scattering process of a photon by the atoms of the medium. For this we consider a thin slab of the medium of thickness $d x^{\prime}$ extending in the $y-z$ plane positioned at $x=x^{\prime}$. As will be shown in the Appendix, the contribution of all the possible ways that a photon arrives at the detector located at position $(x, 0,0)$ via scattering with the atoms in this slab is given by

$$
d A\left(x^{\prime} ; x, t\right)=A_{0}\left(x^{\prime}, t\right) b e^{i k\left(x-x^{\prime}\right)} d x^{\prime},
$$

where $b$ is some complex constant that is specific to the dielectric medium.

We are now ready to analyze the different scattering contributions. First we consider single scattering contributions in the forward direction $A_{f}$, which we will graphically display by

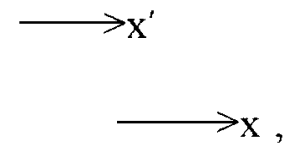

where the scattering occurs somewhere on the plane at $x^{\prime}$ and the detector is located on the $x$ axis at position $x$. We chop the medium into slabs of thickness $d x^{\prime}$ and by using Eqs. (12) and (13) and integrating over all the possible values of $x^{\prime}$ we can write for the resulting amplitude $A_{f}{ }^{9}$

$A_{f}(x, t)=\int_{0}^{x} A_{0}\left(x^{\prime}, t\right) b e^{i k\left(x-x^{\prime}\right)} d x^{\prime}=b x A e^{i(k x-\omega t)}$.

Next we evaluate the contributions due to a single backward scattering. For this we have to sum the contributions of all the possible ways that a photon can be scattered at some position $x^{\prime}$ in order to arrive at the detector at position $x$ at time $t$, where $x^{\prime}>x$. We get

$$
\begin{aligned}
& \multicolumn{1}{c}{} \\
A_{b}(x, t) & =\int_{x}^{\infty} A_{0}\left(x^{\prime}, t\right) b e^{i k\left(x^{\prime}-x\right)} d x^{\prime} \\
& =b A e^{i(-k x-\omega t)} \int_{x}^{\infty} e^{2 i k x^{\prime}} d x^{\prime} \\
& =-\frac{b}{2 i k} A e^{i(k x-\omega t)} .
\end{aligned}
$$

As usual, the upper limit of this integral is taken to be equal to 0 for realistic physical situations. ${ }^{10}$

We now continue with second-order scattering processes. We have the following four contributions (see Fig. 2).

Two forward scattering processes.

Let the first scattering process occur at a position $x^{\prime \prime}$ between $x^{\prime \prime}=0$ and $x^{\prime \prime}=x$ and the second at $x^{\prime}$ with $x^{\prime \prime}<x^{\prime}$ $<x$. Using our result for the amplitude of a single forward scattering we have

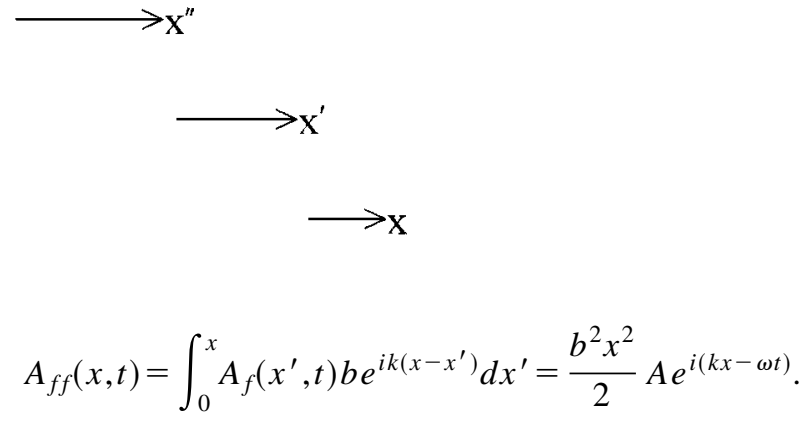

Forward scattering followed by backward scattering yields

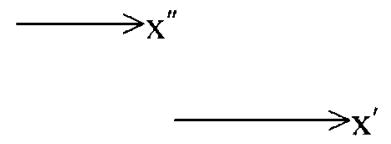

$$
\begin{aligned}
\mathrm{x} & \longleftarrow \\
A_{f b}(x, t) & =\int_{x}^{\infty} A_{f}\left(x^{\prime}, t\right) b e^{i k\left(x^{\prime}-x\right)} d x^{\prime} \\
& =b^{2} A e^{i(-k x-\omega t)} \int_{x}^{\infty} x^{\prime} e^{2 i k x^{\prime}} d x^{\prime} \\
& =\left(\frac{1}{(2 i k)^{2}}-\frac{x}{2 i k}\right) b^{2} A e^{i(k x-\omega t)},
\end{aligned}
$$

where the integral is easily evaluated by integration in parts. $^{11}$

Next, we have the alternative process, backward scattering followed by forward scattering:
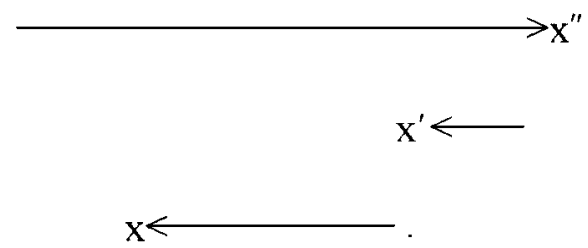

This gives

$$
\begin{aligned}
A_{b f}(x, t) & =\int_{x}^{\infty} A_{b}\left(x^{\prime}, t\right) b e^{i k\left(x^{\prime}-x\right)} d x^{\prime} \\
& =\frac{1}{(2 i k)^{2}} b^{2} A e^{i(k x-\omega t)} .
\end{aligned}
$$

Finally, we have to evaluate the contributions of two backscattering events:

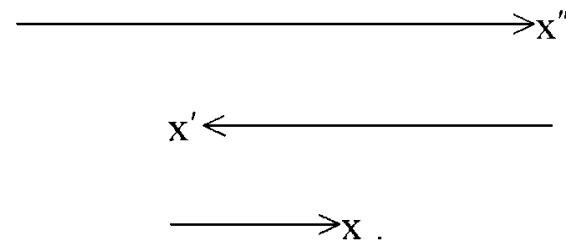


This process can be viewed as a scattering at $x^{\prime}\left(0<x^{\prime \prime}<x\right)$ of a backscattered amplitude. Thus we may write

$$
\begin{aligned}
A_{b b}(x, t) & =\int_{0}^{x} A_{b}\left(x^{\prime}, t\right) b e^{i k\left(x-x^{\prime}\right)} d x^{\prime} \\
& =-\frac{b^{2} x}{2 i k} A e^{i(k x-\omega t)} .
\end{aligned}
$$

This completes all second-order scattering processes.

For the total amplitude we have up to now:

$$
\begin{aligned}
A(x, t)= & A_{0}(x, t)+A_{f}(x, t)+A_{b}(x, t)+A_{f f}(x, t)+A_{b b}(x, t) \\
& +A_{f b}(x, t)+A_{b f}(x, t)=\left[1+\left(\frac{-1}{2 i k}+x\right)\right. \\
& \left.\times b\left(\frac{1}{2(i k)^{2}}-\frac{x}{i k}+\frac{x^{2}}{2}\right) b^{2}\right] A e^{i(k x-\omega t)} .
\end{aligned}
$$

Now let us compare this result with the conventional description given in Eq. (11). Evidently, if we put

$$
b=\frac{i k \chi}{2},
$$

the expansions of Eqs. (11) and (20) are identical:

$$
\begin{aligned}
A(x, t)= & {\left[1+\left(-\frac{1}{4}+\frac{i k x}{2}\right) \chi+\left(\frac{1}{8}-\frac{i k x}{4}+\frac{(i k x)^{2}}{8}\right) \chi^{2}\right.} \\
& \left.+O\left(\chi^{3}\right)\right] A e^{i(k x-\omega t)}
\end{aligned}
$$

Thus we see that (up to second-order scattering processes) we obtain identical results from both points of view. Note that the scattering process, described by the parameter $b$, is proportional to the susceptibility of the medium $\chi$, and that $b$ is purely imaginary.

We can now continue with higher-order scattering processes. If we define the coefficients for the $p$-order scattering process as

$$
A(x, t)=\left[\sum_{p=0}^{\infty} a_{p} b^{p}\right] A e^{i(k x-\omega t)},
$$

we can obtain a general recursion relation between the amplitude due to $p+1$ scattering processes in terms of the amplitude of $p$ scattering processes. This can be done by first dividing the amplitudes for $p$ scattering processes into paths that arrive from positive and negative $x$ directions, respectively. Then we add one extra forward and one extra backward scattering process to these paths, yielding four integrals. Summation leads to the result:

$$
a_{p+1}(x)=b \int_{0}^{x} a_{p}\left(x^{\prime}\right) d x^{\prime}+b e^{-2 i k x} \int_{x}^{\infty} a_{p}\left(x^{\prime}\right) e^{2 i k x^{\prime}} d x^{\prime} .
$$

Application of this recursion relation yields for the next term $(p=3)$ in the expansion:

$$
\left(-\frac{5}{64}+\frac{5 i k x}{32}-\frac{3(i k x)^{2}}{32}+\frac{(i k x)^{3}}{48}\right) \chi^{3},
$$

in agreement with Eq. (11). Using the method described in Ref. 5 a general proof can be given that the recursion relation is in agreement with Eq. (11).

\section{REFLECTION}

We could obtain the results for the amplitude of reflection using the approach of the previous section, but we can get the results more easily as follows. The treatment given above included forward scattering as well as backscattering of photons. Therefore, we should obtain the equations for reflection from the previous section if we simply evaluate the scattered amplitudes for $x=0$ and omit the amplitude of the unscattered photon. Since all the contributions obtained in this way are due to paths that arrive at $x=0$ from positive $x$ values, the resulting amplitudes all propagate further in the negative $x$ direction without any scattering afterwards. We thus get from the result of the previous section:

$$
A_{r}(x, t)=\left[-\frac{1}{4} \chi+\frac{1}{8} \chi^{2}-\frac{5}{64} \chi^{3}+O\left(\chi^{4}\right)\right] A e^{i(-k x-\omega t)} .
$$

The conventional EM treatment gives for the reflected light: ${ }^{7}$

$$
E_{r}(x, t)=\frac{1-n}{n+1} E_{0} e^{i(-k x-\omega t)} .
$$

Expanding this in terms of $\chi$ using Eq. (10) we obtain

$$
E_{r}(x, t)=\left[-\frac{1}{4} \chi+\frac{1}{8} \chi^{2}-\frac{5}{64} \chi^{3}+O\left(\chi^{4}\right)\right] E_{0} e^{i(-k x-\omega t)} .
$$

Thus for reflection, too, the results of both treatments are exactly the same, as they should be. An important difference between the treatments is, however, that in the usual electromagnetic approach the reflection is taken to be caused only by the surfaces of the dielectric, whereas in the scattering approach we clearly see that all the atoms in the medium contribute to the reflection.

It is interesting to note that the arguments used in the beginning of this section are equivalent to the statement in the electromagnetic theory that at the boundary the electric field must be continuous.

\section{PHOTON PROPAGATION IN A TRANSPARENT MEDIUM OF FINITE LENGTH}

We now apply the formalism to transmission and reflection at a transparent plate with a refractive index $n$ for light incident normal to the plate. Let the plate be positioned between $x=0$ and $x=L$, and oriented normal to the $x$ axis (see Fig. 3). The only difference from the preceding sections is that now the upper limit of the integrals involving backscattering are restricted to $x=L$.

For the analysis it is helpful to evaluate first the amplitude somewhere in the medium due to a single forward scattering, $A_{f}(x)$, and due to a single backwards scattering, $A_{b}(x)$. Leaving out the exponential time factor that, as we have seen above, is common to all terms when we use monochromatic light, we find:

$$
\begin{aligned}
A_{f}(x) & =A e^{i k x} \frac{i k \chi}{2} \int_{0}^{x} d x^{\prime}=A e^{i k x} \frac{i k \chi^{x}}{2}, \\
A_{b}(x) & =\frac{i k \chi}{2} \int_{x}^{L} A e^{i k x^{\prime}} e^{i k\left(x^{\prime}-x\right)} d x^{\prime} \\
& =A e^{i k x} \frac{\chi}{4}\left(e^{2 i k(L-x)}-1\right) .
\end{aligned}
$$




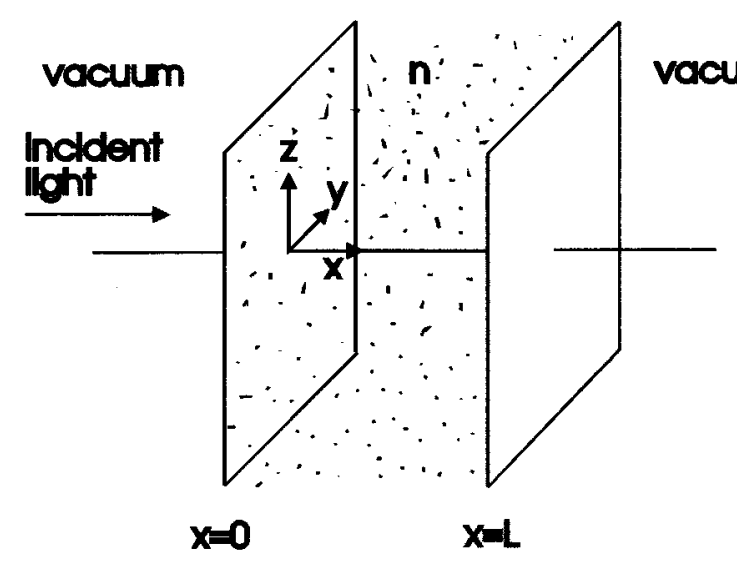

Fig. 3. Light is normally incident on a slab of thickness $L$.

With these amplitudes we can now evaluate the first few terms for transmission and reflection. For transmission we have:

$$
\begin{aligned}
A_{0}(L) & =A e^{i k L}, \\
A_{f}(L) & =A e^{i k L} \frac{i k L}{2} \chi, \\
A_{f f}(L) & =\frac{i k \chi}{2} \int_{0}^{L} A_{f}\left(x^{\prime}\right) e^{i k\left(L-x^{\prime}\right)} d x^{\prime} \\
& =-A e^{i k L} \frac{k^{2} L^{2}}{8} \chi^{2}, \\
A_{b b}(L) & =\frac{i k \chi}{2} \int_{0}^{L} A_{b}\left(x^{\prime}\right) e^{i k\left(L-x^{\prime}\right)} d x^{\prime} \\
& =A e^{i k L}\left(-\frac{1}{16}-\frac{i k L}{8}+\frac{1}{16} e^{2 i k L}\right) \chi^{2} .
\end{aligned}
$$

This yields for the total transmission amplitude up to second order in $\chi$ :

$$
\begin{aligned}
A_{t}(L)= & A e^{i k L}\left[1+\frac{i k L}{2} \chi+\left(-\frac{1}{16}-\frac{i k L}{8}-\frac{k^{2} L^{2}}{8}\right.\right. \\
& \left.\left.+\frac{1}{16}\right) \chi^{2}+O\left(\chi^{3}\right)\right] .
\end{aligned}
$$

For reflection we find contributions:

$$
\begin{aligned}
A_{b}(0) & =\frac{\chi}{4} A\left(e^{2 i k L}-1\right), \\
A_{f b}(0) & =A_{b f}(0)=\frac{i k \chi}{2} \int_{0}^{L} A_{f}\left(x^{\prime}\right) e^{i k x^{\prime}} d x^{\prime} \\
& =\left(\frac{1}{16}-\frac{1}{16} e^{2 i k L}+\frac{i k L}{8} e^{2 i k L}\right) A \chi^{2} .
\end{aligned}
$$

So that up to second order in $\chi$ we have for reflection:

$$
\begin{aligned}
A_{r}= & \left(\frac{1}{4} e^{2 i k L}-\frac{1}{4}\right) A \chi+\left(\frac{1}{8}-\frac{1}{8} e^{2 i k L}\right. \\
& \left.+\frac{i k L}{4} e^{2 i k L}\right) A \chi^{2}+O\left(\chi^{3}\right) .
\end{aligned}
$$

Classical electromagnetic analysis of this problem yields the result. $^{12}$

$$
E_{t}=E_{0} e^{i n k L} \frac{1-r^{2}}{1-r^{2} e^{2 i n k L}}
$$

and

$$
E_{r}=E_{0} \frac{r-r e^{2 i n k L}}{1-r^{2} e^{2 i n k L},}
$$

where

$$
r=\frac{1-n}{n+1}
$$

Expanding these results in terms of $\chi$ gives results identical to the treatment given above.

Once more we see that all the molecules in the slab contribute, in principle, to the reflection, but the results are equivalent to the treatment where only the boundaries are thought to contribute to reflection.

Although the analysis given here assumes a plane incoming wave, the results will hold also for realistic beams, e.g., a Gaussian laser beam of diameter $D$, provided that the divergence of the beam over a distance $L$ is neglectible which requires that $L \lambda / D^{2} \ll 1$. In practice, this means $D \gg \lambda$, so that the Gaussian profile also provides the required behavior for $f(\theta)$ in Eq. (A2).

\section{DISCUSSION}

Let us begin the discussion with a citation of Feynman in his original paper on the path integral formalism: ${ }^{13}$ " $a l$ though it does not yield new results there is pleasure in recognizing old things from a new point of view.' This is, of course, true for the present approach. The main advantage of the description given here is that it deals with the quantum nature of photons: it combines the wave-like properties (resulting in the phase delay) and the particle properties (propagation of single photons) in a simple theory. The path integral formalism very clearly illustrates the essential wonder of quantum mechanics in an appealing way: We only have to consider familiar classical paths, but the strange thing is that if there is no way of telling which path has actually been taken, the particle in a way has taken all the paths.

The essential result of our analysis is that we can interpret the propagation of a photon through a medium as a sum of alternative paths. In examining the exponential term in Eq. (9) one would expect at first sight that it should be expanded in terms of $(n-1)$. Indeed, this is what Feynman suggested. ${ }^{2}$ However, this works only for values of $n$ close to 1 . The fact that we have to expand the exponent in terms of $\chi$ is, from a physical point of view, not really surprising since the essential process involved in the interaction between light and matter, dipole scattering, depends on the polarizability of the medium.

A remarkable outcome of an analysis of this sort is that the resulting amplitude is finite. By adding all possible paths one might fear that the resulting expression for the amplitude would be divergent. Indeed, the individual terms in our expression can be gigantic. As an example, let us consider the situation where the light source is embedded in an infinite medium with refractive index close to 1 , so that we can 
approximate $n-1$ by $\chi / 2$. In that case, using the electromagnetic description the oscillating part of the electric field can be expanded in terms of $\chi$ as:

$$
\begin{aligned}
e^{i k(n-1) x} & =\sum_{p=0}^{\infty} \frac{(i k x(n-1))^{p}}{p !} \\
& =\sum_{p=0}^{\infty} \frac{(i k x(\chi / 2))^{p}}{p !}=\sum_{p=0}^{\infty} \frac{(b x)^{p}}{p !},
\end{aligned}
$$

which according to our new interpretation indicates that the process can be described by forward scattering processes only, since it is easy to show that the amplitude of $p$ forward scattering processes is given by the terms in the summation. (On the average, the amplitudes of backward scattering processes cancel for continuous media.) For values of $x \gg 1 / k$ the largest term in this expansion is obtained for $p=-i b x$. Even for relatively small values of $x$, say $1 \mathrm{~mm}$ and $n$ $=1.01$, this yields a number equal to $125.6^{125.6} / 125.6$ !, which is about $1.25 \times 10^{53}$ for visible light with a wavelength of $500 \mathrm{~nm}$. We are saved only by the fact that these terms oscillate so rapidly that the net result is less than 1 . However, this makes it difficult or impossible to treat the expansion in a perturbative approach where we could limit the analysis to those terms that contribute significantly to the total amplitude.

In deriving our results for the amplitude to detect a photon propagating through a refractive medium, we paid no attention to the normalization. As it turns out, our equations for the amplitude are the same as those for the complex electric field. Therefore, normalization of the amplitudes to conserve the total probability for detecting a photon is equivalent to the normalization of intensity in the electromagnetic case. If we insist that the probability of detecting a photon in time interval $d t$ in an area $O$ should be given by the square of the modulus of the amplitude, we obtain the correct results by generalizing Eq. (7) and replacing $\epsilon_{0}$ by $\epsilon$.

It should be noted that in the above treatment we determined the phase velocity of light. To determine the velocity with which a light pulse propagates through the medium we could follow the usual treatment. ${ }^{14}$ In short, the pulse is decomposed into its Fourier components. Without dispersion, each component travels with the reduced speed $c / n$, so that the pulse also travels with this speed. In a dispersive medium, the pulse will propagate with the group velocity. Although simple and elegant, a treatment similar to the treatment given above, where the time delay of the different paths is taken into account, would be desirable from a didactic point of view. Moreover, the simple Fourier approach yields wrong results for regions with anomalous dispersion ${ }^{15}$ and no distinction is made between signal and group velocity (see also Ref. 16 and references therein for a discussion of the meaning of different velocities of light). A treatment along the lines outlined above should enlighten this more; as an example, the so-called front velocity mentioned by Brillouin is evidently caused by the unscattered photons.

Finally, we come back to the original question about Snell's law. ${ }^{1}$ Our result suggests that we can describe the propagation of photons through a transparent medium with an effective action proportional to the time of transit from source to detector along a straight line path, but with "effective" speed $c / n$ in the transparent medium. Now in order to find the amplitude to go from one point in medium $\mathrm{A}$ to another point in a different medium $\mathrm{B}$, we have to sum the amplitudes of all the possible ways. Following the usual argument in discussing path integrals, ${ }^{6}$ we now conclude that only those paths for which the exponents are stationary give contributions that are not canceled by neighboring paths. ${ }^{17}$ The condition for this to occur is just equivalent to Fermat's principle, from which Snell's law is obtained directly. It should be noted, however, that in this reasoning we assumed that the effective action formula also holds for the case that the boundary of the medium is not perpendicular to the propagation direction of the light. This remains to be proven. For this case, too, it would be nice to evaluate the problem by summing up the scattering amplitudes directly, eventually leading to Ewald-Oseen's extinction theorem. ${ }^{18}$ For another approach to a quantum mechanical description of Snell's law and the Ewald-Oseen theorem the reader is referred to Cook and Milonni. ${ }^{19}$

\section{ACKNOWLEDGMENTS}

I would like to thank Frits W. Wiegel and Dik Feil for a critical reading of the manuscript and valuable discussions, and Bert Altenburg for getting me interested in the problem of photon propagation.

\section{APPENDIX}

The treatment given below serves as a heuristic derivation of the amplitude for scattering by an infinitesimal slab of dielectric material. The situation described macroscopically with Eq. (9) will be treated microscopically with the linear electric dipole approximation for the interaction between light and atoms. The validity of this approximation is discussed in Ref. 20. Furthermore, it is assumed that the frequency of the light is much smaller than the atomic excitation frequencies, which is the condition of Rayleigh light scattering. In this approximation the interaction between atoms and the electromagnetic field only occurs at the position of the atoms, and the interaction consists of annihilation of a photon from the field followed instantaneously by the creation of a photon of the same frequency with a dipole angular distribution. ${ }^{20,21}$ Finally, we will assume that the medium is isotropic.

Consider a thin slab of material positioned at $x^{\prime}$ with thickness $d x^{\prime}$ extending parallel to the $y-z$ plane (Fig. 4). The amplitude for an undisturbed photon to arrive at position $x^{\prime}$ is given by Eq. (3). We will now derive a relation for the amplitude that a photon is scattered by the atoms in the slab toward the detector placed at $(x, 0,0)$. The scattering process is modeled as follows: There is an amplitude that a photon will be absorbed (annihilated) by an atom and that subsequently a new photon is emitted in a new direction. For the present purpose we can limit ourselves to linear elastic dipole scattering. Then the amplitude of the emitted (scattered) photon is proportional to the amplitude of the incoming photon (linear) and it has the same energy as the incoming photon (elastic). We also restrict ourselves to nonresonant interactions, which means that the interaction is instantaneous. The amplitude to scatter in a direction making an angle $\theta$ with the $x$ axis will be dependent on that angle. Let this dependency be described by the function $f(\theta)$ with $f(0)$ $=1$. Integrated over a closed spherical surface centered around the scattering atom, the probability to detect the scattered photon must be constant, independent of the radius of 


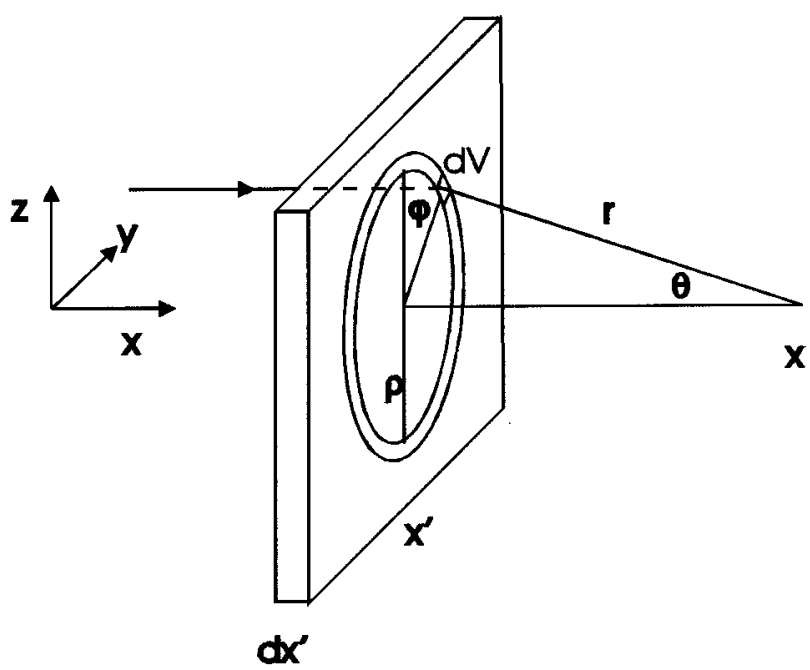

Fig. 4. Calculating the amplitude due to scattering off an infinitesimal thin sheet of dielectric material.

the sphere. Therefore, the amplitude to detect the photon at position $(x, 0,0)$ should be proportional to the inverse of $r$, the distance between the scattering atom and the detector.

We first evaluate the contribution for an infinitesimal volume element $d V$. Within this volume the possible indistinguishable alternatives are proportional to the number of atoms in this volume, and thus the amplitude will be proportional to $d V$ [using postulate (3)]. Since we assumed the amplitudes to be scalar, the contribution of the molecules in the ring with radius $\rho$ (see Fig. 4) is obtained by integrating over $\phi$ :

$$
\begin{aligned}
d A & =2 \pi \rho \frac{f(\theta)}{r} a A_{0}\left(x^{\prime}, t-r / c\right) d \rho d x^{\prime} \\
& =2 \pi \rho \frac{f(\theta)}{r} a A e^{i\left(k\left(x^{\prime}+r\right)-\omega t\right)} d \rho d x^{\prime},
\end{aligned}
$$

where $a$ is a constant for the material considered, that defines the scattering amplitude of a unit volume of the material for $\theta=0$. We have used postulate (6) and (7). Using the fact that $\rho d \rho=r d r$ we obtain

$$
\begin{aligned}
d A\left(x^{\prime} ; x, t\right) & =2 \pi a A e^{i\left(k x^{\prime}-\omega t\right)} \int_{x-x^{\prime}}^{\infty} e^{i k r} f(\theta) d r d x^{\prime} \\
& =-2 \pi a A e^{i\left(k x^{\prime}-\omega t\right)} \frac{e^{i k\left(x-x^{\prime}\right)}}{i k} d x^{\prime} .
\end{aligned}
$$

The integral can be evaluated in this way provided that $f(\theta)$ gradually decreases when $\theta$ increases from 0 to 90 $\operatorname{deg}^{22}$ and that $x-x^{\prime} \gg \lambda$. If we collect the constants in the above equation into a new constant $b=2 \pi i a / k$, we obtain the relation used in the text:

$$
\begin{aligned}
d A\left(x^{\prime} ; x, t\right) & =A e^{i\left(k x^{\prime}-\omega t\right)} b e^{i k\left(x-x^{\prime}\right)} d x^{\prime} \\
& =A_{0}\left(x^{\prime}, t\right) b e^{i k\left(x-x^{\prime}\right)} d x^{\prime} .
\end{aligned}
$$

The formula was derived for forward scattering. For backscattering the argument in the exponential is $i k\left(x^{\prime}-x\right)$.

Thus the difference in phase between the amplitude for the direct (unscattered) path and the total amplitude for all paths that have scattered once is only given by the phase of the constant $b$. As will become clear from Eq. (21), $b$ is purely imaginary, which yields a phase delay of $90 \mathrm{deg}$ for the scattered contribution. Ultimately, this phase delay accounts for the reduced phase velocity. This analysis clearly illustrates the relation between path integral formalism and Huygens principle. ${ }^{13}$ The fact that $b$ is purely imaginary is in agreement with the required phase difference between the nonscattered and scattered contributions of $\pi / 2$ (see Ref. 7, p. 438). The function $f(\theta)$ plays the same role as the obliquity factor (see Ref. 7, p. 434).

The evaluation of Eq. (A2) given here is only valid in the limit $x-x^{\prime} \gg \lambda$ so that the integrand has made a sufficient number of oscillations before it attenuates. For smaller values of $x-x^{\prime}$ the exact formula of $f(\theta)$ should be used, which would require one to take into account the polarization of the light. An alternative approach is to calculate the electric field due to the scattering of the molecules in the slab using the electromagnetic approach outlined by James and Griffiths. ${ }^{5}$ Their result—Eq. (20)—can be rewritten as

$$
d E=\frac{i k \chi}{2} e^{i k\left(x-x^{\prime}\right)} E(x, t) d x^{\prime} .
$$

If we now interpret this complex field to be proportional to the amplitude, as we did in Sec. II, this result is in agreement with (A3). Moreover, we have thus obtained the identification between $b$ and $\chi$ [Eq. (21)] immediately. It should be noted that with this approach the polarization of the light is treated correctly. The essential restriction in the model of James and Griffiths is that the polarization is linear to the electric field and has no phase delay with respect to the oscillating field. This is equivalent to the condition of linear elastic dipole scattering for which the fully quantum mechanical treatment gives identical results as the classical description. $^{21}$

${ }^{1}$ Steve Blau and Brad Halfpap, "Question \#21. Snell's law in quantum mechanics,” Am. J. Phys. 63(7), 583 (1995).

${ }^{2}$ Richard P. Feynman, QED, the Strange Theory of Light and Matter (Princeton U.P., Princeton, 1985).

${ }^{3}$ Richard P. Feynman, The Feynman Lectures on Physics (Addison-Wesley, Reading, MA, 1963), Part I, Sec. 26-6.

${ }^{4}$ John A. Wheeler, "The young Feynman," Phys. Today 42(2), 24-28 (1989).

${ }^{5}$ Mary B. James and David J. Griffiths, "Why the speed of light is reduced in a transparent medium," Am. J. Phys. 60(4), 309-313 (1992).

${ }^{6}$ Richard P. Feynman and A. R. Hibbs, Quantum Mechanics and Path Integrals (McGraw-Hill, New York, 1965). For further interpretation of amplitudes see also the first chapters in the Feynman lectures Part III (Ref. 3).

${ }^{7}$ Eugene Hecht, Optics (Addison-Wesley, Reading, MA, 1987), 2nd ed.

${ }^{8}$ John David Jackson, Classical Electrodynamics (Wiley, New York, 1975), 2nd ed.

${ }^{9}$ Technically, some of the integration regions in Eq. (14)-(19) violate the condition $x-x^{\prime} \gg \lambda$ needed for the evaluation of Eq. (A2) and therefore (A3) given in the Appendix to be valid. In view of the alternative approach indicated in the Appendix, however, use of Eq. (A3) seems to be justified for all values of $x-x^{\prime}$.

${ }^{10}$ See Ref. 3, Sec. 30-7. Physically this term is due to scattering contributions at $x$ approaching infinity. The term disappears if we take into account that the light was turned on in the distant past or we account for a small loss in the material. The problem can be mathematically handled by introducing an attenuation term $\exp (-\varepsilon x)$ in the integral where $\varepsilon$ can be taken arbitrarily small but finite. See also the notes in Ref. 5 .

${ }^{11}$ In the evaluation of this integral we obtain a value that is formally equal to $\infty \exp (i \infty)$. Again this term disappears for realistic physical situations using the reasoning in Ref. 10. 
${ }^{12}$ Reference 7, p. 366.

${ }^{13}$ Richard P. Feynman, "Space-Time Approach to Non Relativistic Quantum Mechanics,"' Rev. Mod. Phys. 20, 367-387 (1948). A reprint of this paper can be found in: Julian Schwinger, Selected Papers on Quantum Electrodynamics (Dover, New York, 1958).

${ }^{14}$ Reference 7, p. 252.

${ }^{15}$ Leon Brillouin, Wave Propagation and Group Velocity (Academic, New York, 1960).

${ }^{16}$ S. C. Bloch, "Eighth Velocity of Light," Am. J. Phys. 45(6) 538-549 (1977).

${ }^{17}$ Reference 3, Chap. 26; Ref. 2.

${ }^{18}$ Max Born and Emil Wolf, Principles of Optics (Pergamon, New York, 1980), 6th ed., Sec. 2.4.2.

${ }^{19}$ R. J. Cook and P. W. Milonni, "Quantum theory of an atom near partially reflecting walls," Phys. Rev. A 35(12) 5081-5087 (1987). In this article the interaction between a single atom and a layer of atoms is obtained by starting from the Schrödinger equation. The treatment is limited to one dimension and single backscattering events. As the material of the present article is basically concerned with the contributions of multiscattering in three dimension, the combination will yield a fully quantum mechanical description, including source-related effects.

${ }^{20}$ Rodney Loudon, The Theory of Light (Clarendon, Oxford, 1983), 2nd ed., Sec. 5.3.

${ }^{21}$ Reference 20, p. 316.

${ }^{22}$ Reference 3, Sec. 30-7. In the case of "scalar" dipole scattering, $f(\theta)$ $=\cos \theta$. In reality, polarization effects will result in a somewhat steeper decay to 0 as $\theta$ approaches $90 \mathrm{deg}$. In any case the effect of $f(\theta)$ is to taper off the contributions to the integral for large values of $r$, as discussed in Ref. 3. 